\title{
Typicality-Based Variational Cluster Approach to Thermodynamic Properties of the Hubbard Model
}

\author{
Hisao Nishida ${ }^{1}$, Ryo Fujiuchi ${ }^{1}$, Koudai Sugimoto ${ }^{2}$, and Yukinori Ohta ${ }^{1}$ \\ ${ }^{1}$ Department of Physics, Chiba University, Chiba 263-8522, Japan and \\ ${ }^{2}$ Department of Physics, Keio University, Yokohama 223-8522, Japan
}

(Dated: September 23, 2019)

\begin{abstract}
We develop the variational-cluster-approximation method based on the thermal-pure-quantumstate approach and apply the method to the calculations of the thermodynamic properties of the Hubbard model, thereby obtaining the temperature dependence of the single-particle excitation spectra, entropy and specific heat, and order parameter of the antiferromagnetic phase efficiently. We thus demonstrate that the method developed here has a potential power for elucidating finitetemperature properties of strongly correlated electron systems.
\end{abstract}

Calculation of finite-temperature properties of strongly correlated quantum systems, for which perturbation theory breaks down, is a hard and challenging task because many excited states must be taken into account for evaluation of their physical quantities. In the grand canonical theory, we need to know all the eigenvalues $E_{\nu}$ and corresponding eigenstates $|\nu\rangle$ of the relevant Hamiltonian to evaluate the ensemble average of an operator $\hat{O}$ at temperature $T$, which is given as

$$
\langle\hat{O}\rangle_{\beta, \mu}^{\mathrm{ens}}=\frac{1}{Z(\beta, \mu)} \sum_{\nu} e^{-\beta\left(E_{\nu}-\mu N_{\nu}\right)}\langle\nu|\hat{O}| \nu\rangle,
$$

where $\beta=1 / T$ is the reciprocal temperature, $\mu$ is the chemical potential, $Z(\beta, \mu)=\sum_{\nu} e^{-\beta\left(E_{\nu}-\mu N_{\nu}\right)}$ is the grand partition function, and $N_{\nu}$ is the number of particles of the state $|\nu\rangle$. A straightforward way to solve this problem is to fully or partially diagonalize the Hamiltonian. Although for quantum lattice models one can in principle evaluate this average if the system size is small enough, one immediately encounters a severe restriction because the dimension of the Hilbert space grows rapidly as the system size increases.

In this respect, quantum cluster approximations based on the self-energy functional theory [1, 2] are found to be useful for treating the systems in the thermodynamic limit, the techniques of which include the dynamical cluster approximation, the single-site or cellular dynamicalmean-field theory (DMFT), the cluster perturbation theory $(\mathrm{CPT})$, and the variational cluster approach (VCA). The DMFT combined with the quantum Monte Calro (QMC) method for a single-impurity solver [1, 3, 4] is often used to obtain the electronic states at finite temperatures. However, it is well-known that the QMC method involves the notorious negative-sign problem [5, 6], especially in itinerant fermionic systems.

Recently, the thermal-pure-quantum (TPQ) state approach based on the typicality has been found to be useful for obtaining physical quantities at finite temperatures 7 9]. Based on the typicality, i.e., the fact that almost all the pure quantum states on an energy shell are equilibrium states [10 13], one can construct the TPQ state by lowering temperature, starting from a random vector corresponding to a thermal equilibrium state at infinite temperature [7 9 ]. Some pioneering work [1418] also uses the random vector to obtain the thermal average. Thus, the ensemble average at a finite temperature may be calculated by a single TPQ state obtained from the imaginary-time evolution of the random vector, instead of calculating all the relevant eigenstates and summing over the average values multiplied by the Boltzmann weight, as in Eq. (11). Thereby, the computational cost can be reduced drastically in this approach although one needs to take a random-sampling average over the TPQ states. Another advantage of this approach is that there are no negative-sign problems occurring in the QMC method. Moreover, because one needs not diagonalize the huge Hamiltonian matrix, one can treat the larger-size systems. We should also emphasize that one can obtain not only the static thermal quantities but also the dynamical quantities such as the excitation spectrum using the typicality-based analyses $19-26$.

In this paper, we calculate the finite-temperature properties of the Hubbard model, a representative of strongly correlated electron models, by making use of the TPQstate approach. First, we use this approach in the CPT 27, 28] and calculate the single-particle excitation spectra in the thermodynamic limit. We then use this approach in the VCA 29 32] and calculate the grand potential as a function of the symmetry-breaking Weiss field for antiferromagnetism. We moreover calculate the static thermal quantities, such as the entropy and specific heat of the system. In a previous study [33], the same model as ours was investigated by the VCA, where the block Lanczos method was used as a quantum cluster solver. There, the calculations of the single-particle Green's functions for all eigenstates with nonvanishing Boltzmann weight are required, and therefore the computational cost rapidly rises up at high temperatures. On the other hand, the method presented here requires the Green's functions for a single TPQ state, thereby reducing the computational cost drastically. We thus demonstrate that the TPQ-state approach provides a powerful technique for elucidating the thermodynamic properties 
of the Hubbard model even at high temperatures.

In what follows, we first introduce the TPQ-state formalism briefly, and then present the results obtained by the CPT and VCA combined with the TPQ-state approach. We consider the single-band Hubbard model defined by the Hamiltonian

$$
\hat{\mathcal{H}}=-t \sum_{\langle i, j\rangle} \hat{c}_{i, \sigma}^{\dagger} \hat{c}_{j, \sigma}+U \sum_{i} \hat{n}_{i, \uparrow} \hat{n}_{i \downarrow},
$$

where $\hat{c}_{i, \sigma}^{\dagger}\left(\hat{c}_{i, \sigma}\right)$ is the creation (annihilation) operator of an electron at site $i$ with spin $\sigma, \hat{n}_{i, \sigma}=\hat{c}_{i, \sigma}^{\dagger} \hat{c}_{i, \sigma}$ is the electron number operator, $t$ is the hopping integral, and $U$ is the on-site Coulomb interaction. $\langle i, j\rangle$ represents the nearest-neighbor pair of sites. We restrict ourselves to the cases of the one-dimensional chain and two-dimensional square lattice at half filling.

To construct the grand-canonical TPQ state, we prepare a random vector

$$
\left|\psi_{0}\right\rangle=\sum_{x=1}^{N_{\mathrm{F}}} c_{x}|x\rangle
$$

where $|x\rangle$ is the orthogonal basis of the Hamiltonian, $N_{\mathrm{F}}=\sum_{n=0}^{2 L_{\mathrm{s}}} \frac{\left(2 L_{\mathrm{s}}\right) !}{n !\left(2 L_{\mathrm{s}}-n\right) !}$ is the dimension of the Fock space, $L_{\mathrm{s}}$ is the number of lattice sites in the system, and $c_{x}$ is a random complex number satisfying $\sum_{x}\left|c_{x}\right|^{2}=1$. This state corresponds to the TPQ state at $\beta=0$ [14], so that the average value at infinite temperature is given by $\langle\hat{O}\rangle_{\beta=0}^{\text {ens }}=\overline{\left\langle\psi_{0}|\hat{O}| \psi_{0}\right\rangle}$, where $\cdots$ is the average over the random samplings.

The unnormalized grand-canonical TPQ state at temperature $\beta$ and chemical potential $\mu$ is given as 9]

$$
\left|\psi_{\beta, \mu}\right\rangle=e^{-\beta \hat{\mathcal{K}} / 2}\left|\psi_{0}\right\rangle
$$

with $\hat{\mathcal{K}}=\hat{\mathcal{H}}-\mu \sum_{i, \sigma} \hat{n}_{i, \sigma}$. Then, instead of Eq. (11), we obtain the ensemble average of the operator $\hat{O}$ as

$$
\langle\hat{O}\rangle_{\beta, \mu}^{\mathrm{ens}}=\frac{\overline{\left\langle\psi_{\beta, \mu}|\hat{O}| \psi_{\beta, \mu}\right\rangle}}{\overline{\left\langle\psi_{\beta, \mu} \mid \psi_{\beta, \mu}\right\rangle}} .
$$

The variance from the average value becomes exponentially small as the number of sites increases [8], which means that the average value obtained from the single TPQ state is equal to the ensemble average in the thermodynamic limit.

The retarded dynamical correlation function of the operators $\hat{A}$ and $\hat{B}$ is given by

$$
\begin{aligned}
G_{\hat{A} \hat{B}}(z) & =-i \int_{0}^{\infty} \mathrm{d} t e^{i z t}\langle\hat{A}(t) \hat{B}(0) \mp \hat{B}(0) \hat{A}(t)\rangle_{\beta, \mu}^{\mathrm{ens}} \\
& =G_{\hat{A} \hat{B}}^{+}(z) \mp G_{\hat{B} \hat{A}}^{-}(z),
\end{aligned}
$$

where $\hat{A}(t)=e^{i \hat{\mathcal{K}} t} \hat{A} e^{-i \hat{\mathcal{K}} t}$ is the Heisenberg representation, and the upper (lower) sign is for bosonic (fermionic)
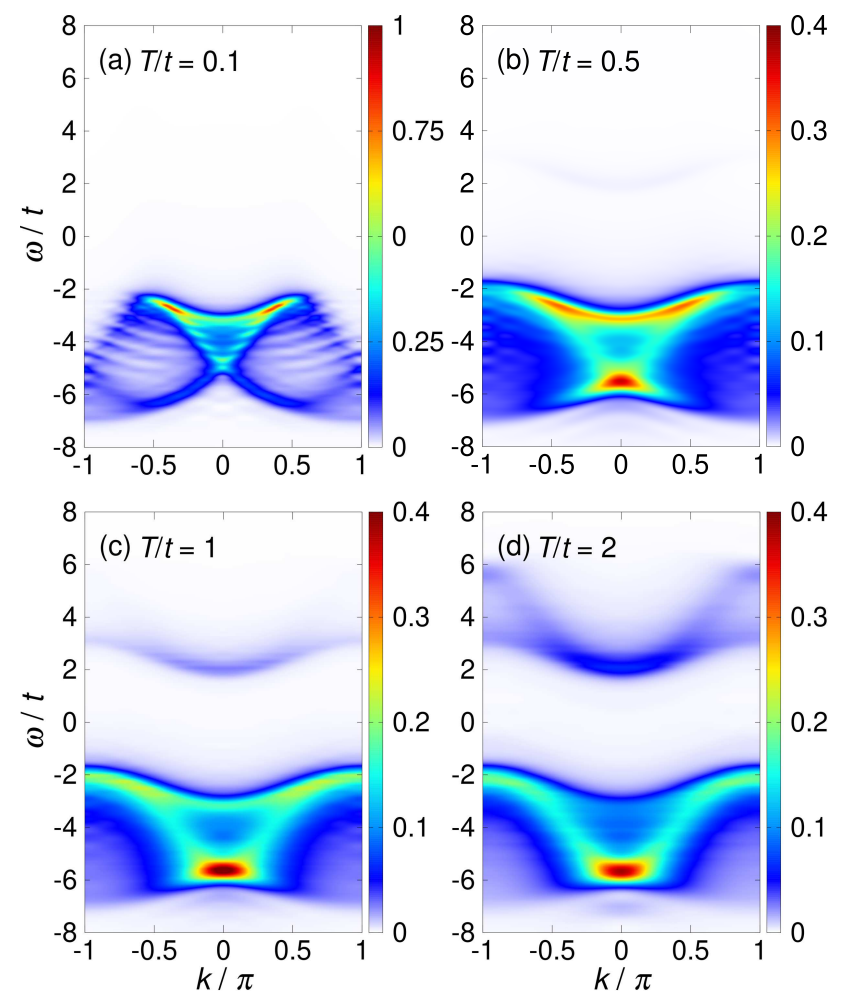

FIG. 1. Contour plot of the photoemission spectra for the onedimensional Hubbard model at (a) $T / t=0.1$, (b) $T / t=0.5$, (c) $T / t=1$, and (d) $T / t=2$ by the CPT. We set $U / t=8$ and assume the size of the cluster to be $L=12$ and the number of Lanzcos iterations to be $N_{\mathrm{L}}=100$. Ten random samplings for the TPQ-state calculations are averaged.

operators. This function is calculated by the Lanczos procedure 16 18]. The single-particle Green's function may then be written as $G_{\hat{c}_{i, a}, \hat{c}_{j, b}^{\dagger}}$, where $a$ and $b$ represent the internal degrees of freedom of the system, such as spin, orbital, sublattice, etc. We abbreviate the singleparticle Green's function as $G_{i a, j b}$.

To calculate the single-particle excitation spectra in the thermodynamic limit, we use the CPT, where the original lattice is divided into the equal-shape disconnected clusters of $L$ sites. We call the collection of the clusters forming the superlattice the reference system, and we denote $\tilde{\boldsymbol{k}}$ as the wave vector in the Brillouin zone defined for the superlattice, i.e., the wave vector is written as $\boldsymbol{k}=\tilde{\boldsymbol{G}}+\tilde{\boldsymbol{k}}$, where $\tilde{\boldsymbol{G}}$ is the reciprocal lattice vector for the superlattice. The Green's function in the CPT is given by [27, 28]

$$
G_{a, b}^{\mathrm{CPT}}(\boldsymbol{k}, z)=\frac{1}{L} \sum_{i, j}^{L} e^{-i \boldsymbol{k} \cdot\left(\boldsymbol{r}_{i}-\boldsymbol{r}_{j}\right)} \tilde{G}_{i a, j b}(\tilde{\boldsymbol{k}}, z)
$$

where $\boldsymbol{r}_{i}$ and $\boldsymbol{r}_{j}$ are the site positions in the cluster and

$$
\tilde{G}(\tilde{\boldsymbol{k}}, z)=\left[\left(G^{\prime}(z)\right)^{-1}-V(\tilde{\boldsymbol{k}})\right]^{-1}
$$



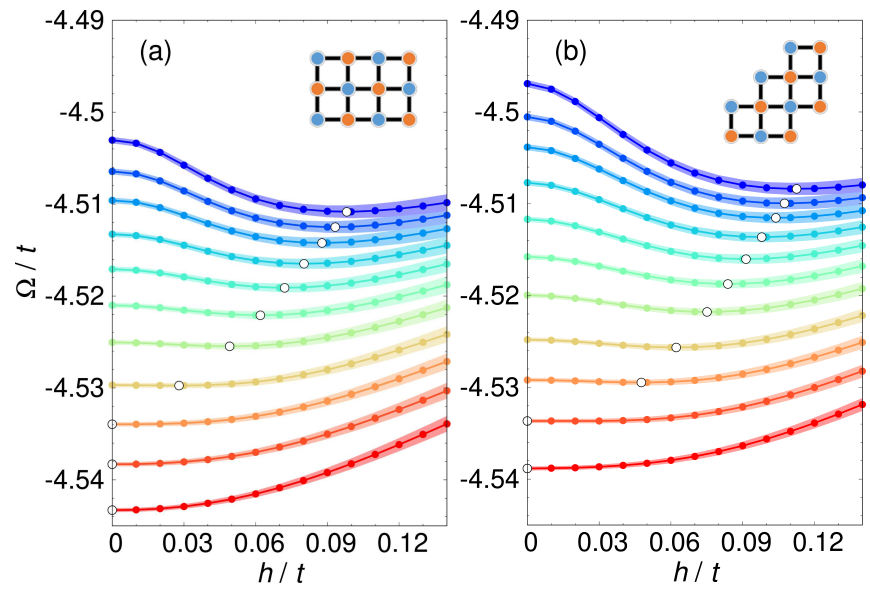

FIG. 2. Calculated grand potentials as a function of the antiferromagnetic Weiss field at temperatures from $T / t=0.2$ (blue line) to 0.3 (red line) in the interval of 0.01 . We assume the square-lattice Hubbard model at $U / t=8$ and set the size of the clusters to be $L=12$ with the shape shown in the inset. The width of the lines indicates the variance coming from the random samplings of the TPQ states. The white open circles represent the points at which the grand potential takes its minimum value. The number of random samplings for the TPQ states is 300 for calculating $\Omega^{\prime}$ and 45 for calculating Green's functions.

is the approximate single-particle Green's function of the original system in the matrix representation. Here, $G^{\prime-1}(z)$ is the inverse of the exact Green's function of the reference system and $V(\tilde{\boldsymbol{k}})$ is the Fourier transform of $V=\left(G^{(0)}\right)^{-1}-\left(G^{(0)}\right)^{-1}$, the difference between the one-body-term Green's functions of the reference and original systems. With the CPT Green's function, the single-particle spectral function is given by $A(\boldsymbol{k}, \omega)=-\frac{1}{\pi} \operatorname{Im} \operatorname{Tr} G^{\mathrm{CPT}}(\boldsymbol{k}, z=\omega+i \eta)$.

First, to demonstrate the validity of the TPQ-state approach combined with the $\mathrm{CPT}$, we calculate the photoemission spectral function $A^{-}(\boldsymbol{k}, \omega)$ for the onedimensional Hubbard model at half filling at various temperatures. The results are shown in Fig. 1, which may be compared with the results obtained by the finitetemperature time-dependent density-matrix renormalization group method [34]. We find that the agreement is very good: At low temperatures, the spinon and holon dispersions caused by the spin-charge separation [35] appear, and with increasing temperature, the spinon dispersion gradually disappears while the holon dispersion remains. Also noticed is that thermally excited electrons go into the upper Hubbard band, leading to the emergence of the spectral weight above the Fermi level. We thus conclude that the TPQ-state approach combined with the CPT indeed works very well.

Next, we discuss the TPQ-state approach combined with the VCA. The VCA is an extended version of the
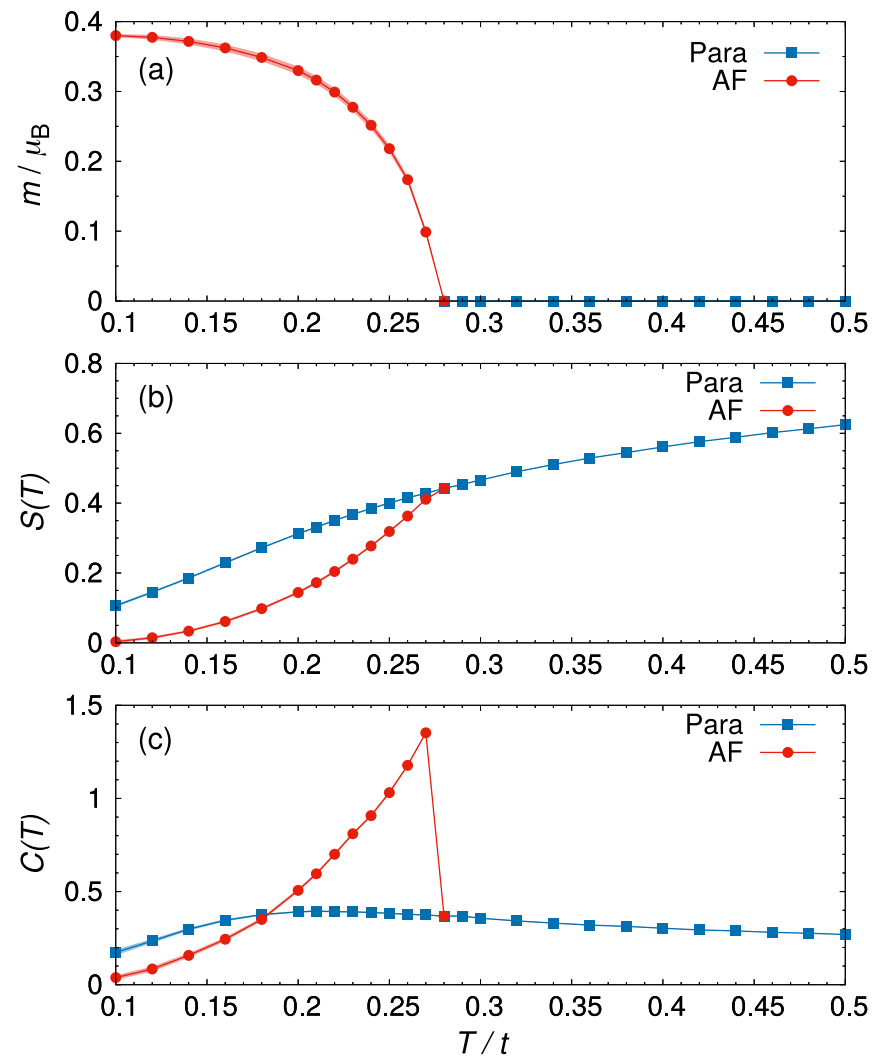

FIG. 3. (a) Calculated order parameter $m$, entropy $S$, and specific heat $C$ as a function of temperature $T$ for the paramagnetic (Para) and antiferromagnetic (AF) phases of the square-lattice Hubbard model at $U / t=8$. We assume the cluster of the size $L=12$ with the shape shown in the inset of Fig. 2(a).

CPT, where the grand potential of the original system is minimized by varying the self-energy of the reference system, or equivalently by varying one-body parameters in the cluster. The grand potential (per site) is given by [31]

$\Omega(\beta)=\Omega^{\prime}(\beta)-\frac{1}{\beta L N_{\mathrm{c}}} \sum_{\tilde{\boldsymbol{k}}} \sum_{n} \ln \operatorname{det}\left[1-V(\tilde{\boldsymbol{k}}) G^{\prime}\left(i \varepsilon_{n}\right)\right]$,

where $N_{\mathrm{c}}$ is the number of the clusters in the system, $\varepsilon_{n}=(2 n+1) \pi / \beta$ is the Matsubara frequency, and

$$
\Omega^{\prime}(\beta)=-\frac{1}{\beta L} \ln \left(N_{\mathrm{F}} \overline{\left\langle\psi_{\beta, \mu} \mid \psi_{\beta, \mu}\right\rangle}\right)
$$

is the grand potential (per site) of the reference system. Here, $\left|\psi_{\beta, \mu}\right\rangle$ is the TPQ state in the cluster. Also, the average value of an operator $\hat{O}$ in the VCA is given by

$$
\langle\hat{O}\rangle=\frac{1}{L N_{\mathrm{c}}} \sum_{\tilde{\boldsymbol{k}}} \sum_{n} \operatorname{Tr}\left\{\hat{O} G^{\prime}\left(i \varepsilon_{n}\right)\left[1-V(\tilde{\boldsymbol{k}}) G^{\prime}\left(i \varepsilon_{n}\right)\right]\right\} .
$$



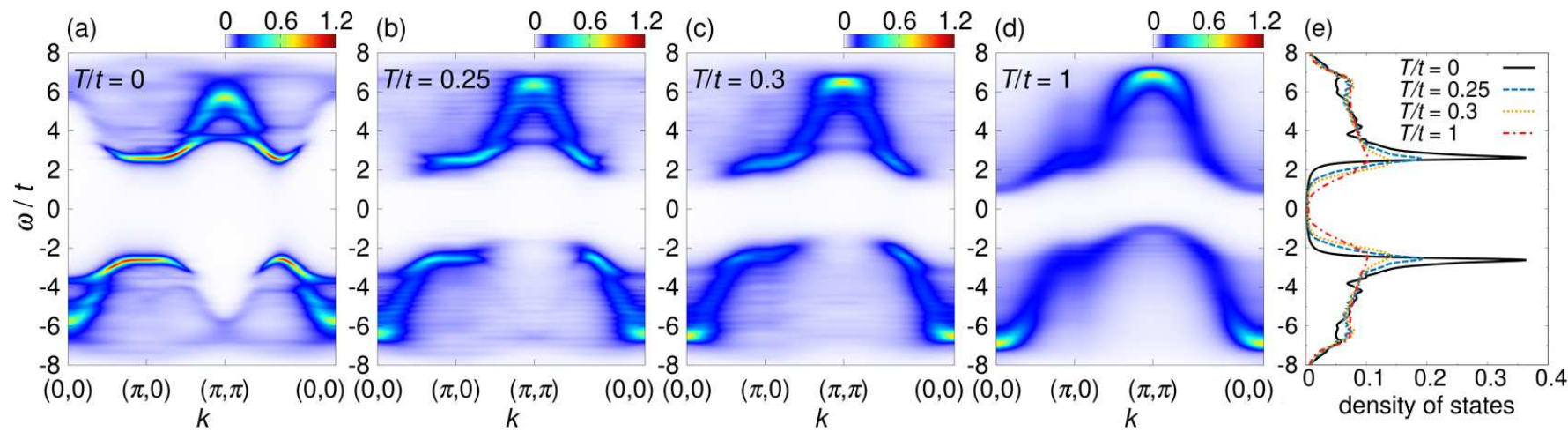

FIG. 4. Contour plot of the calculated single-particle excitation spectra $A(\boldsymbol{k}, \omega)$ for the square-lattice Hubbard model at $U / t=8$ both below and above the antiferromagnetic transition temperature $T_{\mathrm{N}} / t=0.28$. The results are given at (a) $T / t=0$, (b) $T / t=0.25$, (c) $T / t=0.3$, and (d) $T / t=1$. Calculated densities of states at different temperatures are also shown in (e). The cluster size is set to be $L=12$ with the shape shown in the inset of Fig. 2(a). Ten random samplings for the TPQ-state calculations are averaged.

Because Eq. (9) contains the summation over an infinite number of Matsubara frequencies, which is difficult to calculate directly, we replace the summation in the high frequency part by the contour integral in the complex plane, as in Ref. 33 .

The spontaneous symmetry breaking for antiferromagnetism is treated within the framework of the VCA by adding the Weiss field $\hat{\mathcal{H}}^{\prime}=h \sum_{i, \sigma} \sigma e^{i \boldsymbol{Q} \cdot \boldsymbol{r}_{i}} \hat{n}_{i, \sigma}$ to the Hamiltonian of the reference system, where $h$ is the strength of the Weiss field and $\boldsymbol{Q}$ is the ordering vector. The magnetic moment $m=\frac{1}{L} \mu_{\mathrm{B}} \sum_{i, \sigma} \sigma e^{i \boldsymbol{Q} \cdot \boldsymbol{r}_{i}}\left\langle\hat{n}_{i, \sigma}\right\rangle$, where $\mu_{\mathrm{B}}$ is the Bohr magneton, is the order parameter of this system. Figures 2(a) and 2(b) show the grand potentials as a function of the Weiss field for antiferromagnetism with $\boldsymbol{Q}=(\pi, \pi)$, calculated for the square-lattice Hubbard model at half filling with $U / t=8$. We assume two different clusters of size $L=12$ shown in the inset of Figs. 2(a) and 2(b). We find that the grand potentials have a minimum at a finite Weiss field, indicating that the antiferromagnetic ordering actually occurs. The calculated magnetic moment as a function of temperature is shown in Fig. 3(a), where we find that the phase transition is of the second order with the transition temperature $T_{\mathrm{N}} / t=0.28$. We should note here that, according to the Mermin-Wagner-Hohenberg theorem [36, 37], any spontaneous breaking of continuous symmetries is prohibited in two dimension at finite temperature. Our results contradict with this theorem. This is because the clustering of the original lattice cuts off the long-range correlations and correlations beyond the cluster size are treated only in a mean-field level where the Weiss field explicitly breaks the symmetry. Reflecting this situation, the transition temperature decreases as the cluster size increases [33].

An advantage of the VCA is a direct calculation of the thermodynamic function $\Omega(T)$, which enables one to cal- culate the entropy and specific heat of the system directly as $S(T)=-(\mathrm{d} \Omega / \mathrm{d} T)$ and $C(T)=T(\mathrm{~d} S / \mathrm{d} T)$. Figures 3(b) and 3(c) show the results for the temperature dependence of the entropy and specific heat of the system, respectively, calculated for the square-lattice Hubbard model at half filling with $U / t=8$. We observe that a rapid decrease in the entropy occurs below the transition temperature, which is associated with a jump in the specific heat. These behaviors are consistent with the results given in Ref. 33, ensuring that the TPQ-state approach combined with the VCA is a powerful tool for calculating the thermodynamic quantities in systems where any phase transition occurs.

Finally, we calculate the temperature dependence of the single-particle excitation spectra in the TPQ-state approach combined with the VCA, assuming the same model as above. The results are shown in Figs. 4(a)(d), together with the density of states in Fig. 4(e). We find that the energy gap opens both below and above the transition temperature, so that the system is insulating in the entire temperature range. The band dispersions do not change largely across the phase transition point [see Figs. 4(b) and 4(c)]. We also find that the energy gap gradually decreases at very high temperatures because many doublon states are created by thermal excitations. These results demonstrate the behavior of the Mott gap over a wide temperature range.

In summary, based on the typicality, we developed the TPQ-state approach combined with the CPT and VCA and calculated the single-particle excitation spectra and specific heat of the Hubbard model at finite temperatures successfully. The primary advantage of the present approach is that the expectation value of the physical quantity can be obtained from the single TPQ state, so that one can efficiently investigate the thermodynamic properties of the system at high temperatures without diago- 
nalizing the large Hamiltonian matrix. We note that the present approach is not restricted to the Hubbard model but is also applicable to other fermionic as well as bosonic models with strong correlations, and may also be useful for frustrated quantum systems where many nearlydegenerate eigenstates exist at low energies. Moreover, the calculations of the two-body correlation functions by the CPT and VCA [38, 39] can be extended to finite temperatures using the present TPQ-state approach. Our studies thus pave the way for future investigations of strongly correlated quantum systems at finite temperatures.

We thank A. Sugita for tutorial lectures on the typicality-based approaches and T. Yamaguchi for enlightening discussions. This work was supported in part by JSPS KAKENHI Grants (Nos. JP19J20768, JP19K14644, and JP17K05530) and Keio University Academic Development Funds for Individual Research. The numerical calculations were carried out on XC40 at YITP in Kyoto University.

[1] T. Maier, M. Jarrell, T. Pruschke, and M. H. Hettler, Rev. Mod. Phys. 77, 1027 (2005).

[2] D. Sénéchal, arXiv:0806.2690

[3] A. Georges, G. Kotliar, W. Krauth, and M. J. Rozenberg, Rev. Mod. Phys. 68, 13 (1996).

[4] E. Gull, A. J. Millis, A. I. Lichtenstein, A. N. Rubtsov, M. Troyer, and P. Werner, Rev. Mod. Phys. 83, 349 (2011).

[5] E. Y. Loh, Jr., J. E. Gubernatis, R. T. Scalettar, S. R. White, D. J. Scalapino, and R. L. Sugar, Phys. Rev. B 41, 9301 (1990).

[6] M. Troyer and U.-J. Wiese, Phys. Rev. Lett. 94, 170201 (2005).

[7] S. Sugiura and A. Shimizu, Phys. Rev. Lett. 108, 240401 (2012).

[8] S. Sugiura and A. Shimizu, Phys. Rev. Lett. 111, 010401 (2013).

[9] M. Hyuga, S. Sugiura, K. Sakai, and A. Shimizu, Phys. Rev. B 90, 121110(R) (2014).

[10] S. Goldstein, J. L. Lebowitz, R. Tumulka, and N. Zanghì, Phys. Rev. Lett. 96, 050403 (2006).

[11] S. Popescu, A. J. Short, and A. Winter, Nat. Phys. 2, 754 (2006).

[12] A. Sugita, Nonlinear Phenom. Complex Syst.10, 192
(2007).

[13] P. Reimann, Phys. Rev. Lett. 99, 160404 (2007).

[14] M. Imada, and M. Takahashi, J. Phys. Soc. Jpn. 55, 3354 (1986).

[15] A. Hams and H. De Raedt, Phys. Rev. E 62, 4365 (2000).

[16] J. Jaklič and P. Prelovšek, Phys. Rev. B 49, 5065 (1994).

[17] J. Jaklič and P. Prelovšek, Adv. Phys. 49, 1 (2000).

[18] M. Aichhorn, M. Daghofer, H. G. Evertz, and W. von der Linden, Phys. Rev. B 67, 161103(R) (2003).

[19] C. Bartsch and J. Gemmer, Phys. Rev. Lett. 102, 110403 (2009).

[20] T. A. Elsayed and B. V. Fine, Phys. Rev. Lett. 110, 070404 (2013).

[21] R. Steinigeweg, J. Gemmer, and W. Brenig, Phys. Rev. Lett. 112, 120601 (2014).

[22] T. Monnai and A. Sugita, J. Phys. Soc. Jpn. 83, 094001 (2014)

[23] F. Jin, R. Steinigeweg, F. Heidrich-Meisner, K. Michielsen, and H. De Raedt, Phys. Rev. B 92, 205103 (2015).

[24] R. Steinigeweg, J. Herbrych, F. Pollmann, and W. Brenig, Phys. Rev. B 94, 180401(R) (2016).

[25] Y. Yamaji, T. Suzuki, M. Kawamura, arXiv:1802.02854.

[26] J. Richter and R. Steinigeweg, Phys. Rev. B 99, 094419 (2019).

[27] D. Sénéchal, D. Perez, and M. Pioro-Ladrière, Phys. Rev. Lett. 84, 522 (2000).

[28] D. Sénéchal, D. Perez, and D. Plouffe, Phys. Rev. B 66, 075129 (2002).

[29] M. Potthoff, Eur. Phys. J. B 32, 429 (2003).

[30] M. Potthoff, Eur. Phys. J. B 36, 335 (2003).

[31] M. Potthoff, M. Aichhorn, and C. Dahnken, Phys. Rev. Lett. 91, 206402 (2003).

[32] C. Dahnken, M. Aichhorn, W. Hanke, E. Arrigoni, and M. Potthoff, Phys. Rev. B 70, 245110 (2004).

[33] K. Seki, T. Shirakawa, and S. Yunoki, Phys. Rev. B 98, 205114 (2018).

[34] A. Nocera, F. H. L. Essler, and A. E. Feiguin, Phys. Rev. B 97, 045146 (2018).

[35] C. Kim, Z.-X. Shen, N. Motoyama, H. Eisaki, S. Uchida, T. Tohyama, and S. Maekawa, Phys. Rev. B 56, 15589 (1997).

[36] N. D. Mermin and H. Wagner, Phys. Rev. Lett. 17, 1133 (1966).

[37] P. C. Hohenberg, Phys. Rev. 158, 383 (1967).

[38] S. Brehm, E. Arrigoni, M. Aichhorn, and W. Hanke, EPL 89, 27005 (2010).

[39] P. T. Raum, G. Alvarez, T. Maier, and V. W. Scarola, arXiv:1908.10361 